\title{
People analytics effectiveness: developing a framework
}

\author{
Tina Peeters, Jaap Paauwe and Karina Van De Voorde \\ Department of Human Resource Studies, School of Social and Behavioral Sciences, \\ Tilburg University, Tilburg, Netherlands
}

\begin{abstract}
Purpose - The purpose of this paper is to explore the key ingredients that people analytics teams require to contribute to organizational performance. As the information that is currently available is fragmented, it is difficult for organizations to understand what it takes to execute people analytics successfully.

Design/methodology/approach - To identify the key ingredients, a narrative literature review was conducted using both traditional people analytics and broader business intelligence literature. The findings were summarized in the People Analytics Effectiveness Wheel.

Findings - The People Analytics Effectiveness Wheel identifies four categories of ingredients that a people analytics team requires to be effective. These are enabling resources, products, stakeholder management and governance structure. Under each category, multiple sub-themes are discussed, such as data and infrastructure; senior management support; and knowledge, skills, abilities and other characteristics (KSAOs) (enablers).

Practical implications - Many organizations are still trying to set up their people analytics teams, and many others are struggling to improve decision-making by using people analytics. For these companies, this paper provides a comprehensive overview of the current literature and describes what it takes to contribute to organizational performance using people analytics.

Originality/value - This paper is designed to provide organizations and researchers with a comprehensive understanding of what it takes to execute people analytics successfully. By using the People Analytics Effectiveness Wheel as a guideline, scholars are now better equipped to research the processes that are required for the ingredients to be truly effective.
\end{abstract}

Keywords People analytics, HR analytics, Workforce analytics, Organizational performance

Paper type Viewpoint

\section{Introduction}

The human resource management (HRM) function is making steps to combine its intuition, experience and beliefs with the new trend of data analytics (Rasmussen and Ulrich, 2015; Van der Togt and Rasmussen, 2017). Marler and Boudreau define people analytics (data analytics applied to human resources [HR]) as "a HR practice enabled by information technology that uses descriptive, visual, and statistical analyses of data related to HR processes, human capital, organizational performance, and external economic benchmarks to establish business impact and enable data-driven decision-making" (2017, p. 15). People analytics can thus be used to solve pressing business issues, as illustrated, for example, by the people analytics team of ING. The bank was looking to recruit specialists to work on Know Your Customer (KYC). This covers transaction screening; client file enhancement, including documentation and data as well as identity verification; and structural solutions to execute the bank's KYC policies - all ultimately focused on protecting the bank from financial economic crime. However, due to the shortage of people with the necessary skills

(C) Tina Peeters, Jaap Paauwe and Karina Van De Voorde. Published by Emerald Group Publishing Limited. This article is published under the Creative Commons Attribution (CC BY 4.0) licence. Anyone may reproduce, distribute, translate and create derivative works of this article (for both commercial and non-commercial purposes), subject to full attribution to the original publication and authors. The full terms of this licence may be seen at http://creativecommons.org/licences/by/4.0/legalcode
Received 28 April 2020 Revised 28 May 2020 Accepted 6 June 2020

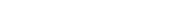

\section{analytics} effectiveness

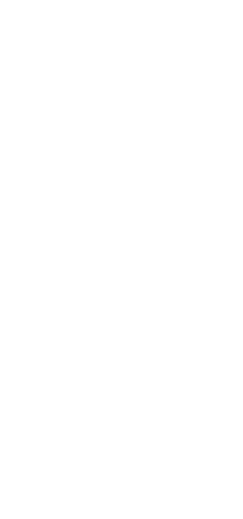


$\underset{7,2}{\text { JOEPP }}$

required for these roles in the global labor market, ING's people analytics team worked to identify which internal ING employees would be suited to fulfill the vacancies at the KYC department. To do so, they matched over 9,000 different job titles to an external database, which afforded them a global overview of the knowledge, skills and abilities of their entire employee population. They were consequently able to determine which employees would fit the profile of the vacancies well, and conversations with those employees ensued. This not only allowed the company to fulfill critical vacancies but also provided internal employees the opportunity to develop themselves into new roles that they may otherwise have never imagined.

Although other companies are also reaping the benefits of people analytics, only $16 \%$ of organizations have actually implemented advanced people analytics in practice (SierraCedar, 2018). This low adaptation rate has caused an academic discussion on the many issues that people analytics is facing in practice (e.g. as reported in a special issue of this journal [Minbaeva, 2017]). Given the growing interest in the area, we believe that the development of a heuristic framework based on the currently available (people) analytics literature is both timely and an important first step to gain a more in-depth understanding of what it takes to establish a successful, advanced analytics team.

By developing this framework on people analytics effectiveness, we contribute to theory as well as practice in three distinct ways. First, although many scholars have discussed key ingredients that are required for a people analytics team in the past (e.g. Andersen, 2016; Green, 2017; Guenole et al., 2017), this information is often fragmented and focused almost exclusively on people analytics. However, considering that other sub-domains of business intelligence fields, such as marketing and customer analytics, are more advanced than people analytics (e.g. Davenport and Harris, 2017; Holsapple et al., 2014), the knowledge from these other sub-domains may provide us with new insights into how to establish an effective people analytics team. We will consequently review both people analytics and business intelligence literature to develop our framework.

Second, while the enablers and (potential) products of a people analytics team have been the focus of various articles (e.g. Green, 2017; Hota and Ghosh, 2013), many questions related to ethics and compliance remain largely unanswered (Van der Laken, 2018). Considering that people data is increasingly regulated by law and the number of ethical questions regarding the usage of people analytics is growing, we believe this to be a lacuna in the people analytics literature. Therefore, this paper also investigates what ingredients should be in place for a people analytics team to achieve both compliance and legitimacy in the eyes of internal and external stakeholders.

Third, we make a contribution to the discussion that is taking place in practice about what it takes for a people analytics team to be successful. While it is, for instance, common practice to evaluate people analytics teams based on their maturity level (e.g. Bersin and Associates, 2012, as cited in Bersin, 2012), the underlying assumption to these models is that the more complex a team's analyses become, the more mature the team is and thus the more value it can add to an organization. However, we believe this assumption is troubling for two reasons. First, as organizations have reported to gain the most added value from their people analytics teams through descriptive analytics (i.e. $80 \%$ at Shell, Van der Togt and Rasmussen, 2017), this linear way of thinking about the maturity level of people analytics appears to be incorrect when the added value of the team is concerned. Second, it is highly likely that more aspects of a team than just the complexity of its analysis affect its potential added value. For instance, while the statistical skills of a team may be excellent, stakeholder knowledge of statistics may be insufficient to understand the insights that the team provides, which causes the team to have limited added value. Therefore, we believe it is time to explore the many different ingredients that can lead to the success of a people analytics team instead of solely the complexity of the analysis. 


\section{Methods}

To identify which ingredients are critical for a people analytics team, we conducted a narrative literature review in two steps. In the first step, we searched for literature relevant to the topic of people analytics effectiveness. We consequently did not review every people analytics or business intelligence paper, but analyzed the most relevant texts in depth. To find relevant articles, we searched for English or Dutch literature using online databases. We specifically used keywords such as "people analytics," "HR analytics," "workforce analytics," "talent analytics" and "business intelligence" and keywords reflecting sub-fields, such as "finance analytics" and "marketing analytics." Furthermore, as people analytics is a relatively new research field, we did not specify a time frame for our search using the first four search terms. For both the business intelligence literature and the sub-fields in particular, we specified that articles needed to be published after 2005. This was done to narrow our search in this relatively older and larger research fields. Thereafter, we employed the snowball technique to find additional relevant literature using the reference list of the literature that we found.

In the second step, we read the search results in detail and assigned codes to each of the ingredients that we encountered that are required for people analytics to be effective. Based on our coding scheme and discussions among the authors of this paper, four different categories of ingredients emerged from the literature: (1) the "enabling resources" of a people analytics team, (2) the "products" the team delivers to the organization, (3) the main "stakeholders" who should be at the receiving end of these products in order to add value to the organization and (4) the "governance structure" that a people analytics team requires to achieve compliance and legitimacy. Based on the literature, as each category appeared to be critical to the success of a people analytics team, we decided to structure our paper around these four categories. Therefore, in the next section, we focus on discussing the ingredients in more detail in relation to the following questions:

(1) What are the enabling resources of a people analytics team?

(2) What types of products should this team deliver to contribute to organizational performance?

(3) Who are the stakeholders of this team, and how should they be managed?

(4) What are the elements of a people analytics team's proper governance structure to safeguard compliance and achieve legitimacy?

\section{The enabling resources of a people analytics team}

In the literature, a number of ingredients can be identified that a people analytics team needs in order to be effective. These are (1) senior management support; (2) data and infrastructure; and (3) the knowledge, skills, abilities, and other characteristics (KSAOs) of people analytics staff (e.g. Andersen, 2016; Green, 2017; Marler and Boudreau, 2017).

Senior management support: According to various scholars (e.g. Davenport and Harris, 2017; Green, 2017; Guenole et al., 2017), support of senior management is one the main prerequisites for an analytical team to be successful. This is because senior management is capable of providing the team with both financial resources and political support. With financial resources, the team can invest in the required equipment, IT infrastructure and people, which together make up the people analytics team. With political support, senior management sends signals to other stakeholders, such as line management and HR professionals, that analytics is important and that data-driven decisions are the future (Davenport and Harris, 2017; Guenole et al., 2017). Guenole et al. (2017) consequently recommend close involvement of the HR director (the highest ranking HR leader of the

\section{People analytics effectiveness}



$\underset{7,2}{\text { JOEPP }}$

company) in a people analytics team and full support from senior leadership outside of the HR function. If such support cannot be achieved, then they warn that the team will likely encounter more resistance from other stakeholders. Internal support from senior management thus appears to be highly important for an effective people analytics team.

\section{Data and IT infrastructure}

Data is at the core of the people analytics function (Davenport and Harris, 2017; Guenole et al., 2017) and is required for a team to conduct analyses and report insights. People analytics may work with various data types, such as traditional HR data (i.e. absenteeism and surveys); business data (i.e. performance) (Edwards and Edwards, 2019); or newer types of data that are, for instance, obtained through personal devices such as location and health (Boudreau and Cascio, 2017). The data that a team uses may exist in various forms, such as structured, unstructured, longitudinal, cross-sectional, qualitative or quantitative (Edwards and Edwards, 2019; Guenole et al., 2017; Holsapple et al., 2014; Van der Laken, 2018).

Although the quantity of data appears to be less of an issue now that much more data than ever before is available from workers (Boudreau and Cascio, 2017), the quality of the data is still a reason that many projects fail, according to Andersen (2017). This is also captured in the popular phrase "garbage in, garbage out" (Andersen, 2017, p. 134), which means that erroneous data will also likely result in erroneous findings. There generally appear to be three major reasons that people analytics teams struggle with low data quality. The first reason is the absence of one overarching database (Barton and Court, 2012), which means that the data the team receives can be out of date. Second, data is often the result of human input, which means that it can be incorrect or incomplete. The third reason is that one concept may have various data definitions in different areas (divisions or subsidiaries) of a business. This can be an especially complex problem once an organization operates in several countries: For example, a full-time workweek is already considered to consist of a different amount of working hours in different countries (e.g. Eurostat, 2013). To cope with the issue of data quality, cleaning the data from errors is considered to be an important, but time-consuming task of a people analytics team (Britnell 2016, as cited in Green, 2017). In practice, this means that a team may spend up to $25-30 \%$ of its time cleaning the data to provide an organization with correct and credible results (Davenport and Harris, 2017).

With regard to IT infrastructure, Marler and Boudreau (2017), among others (e.g. Barton and Court, 2012; Bose, 2009; Trkman et al., 2010), argue that it should be capable of storing and processing a sufficient data quantity and quality and sharing the results with decision-makers. However, this is challenging in many organizations as old IT systems prevent data from being integrated into one system due to siloed information (Barton and Court, 2012) and, as previously mentioned, the absence of shared definitions (Guenole et al., 2017). Although aligning these systems is a time-consuming process, it seems crucial that the IT infrastructure is of high quality for efficiency and the credibility of the results. Furthermore, according to Boudreau and Cascio (2017), it is also important that the right communication channels, techniques and timing are used when sharing information with decision-makers as this can motivate them to act on the insights provided by a people analytics team. Bose (2009) and Boudreau and Cascio (2017) state that the key elements in this regard are that the information provided should be aligned to the business strategy and that easy-to-access information should be delivered to decision-makers on demand. In practice, this means that any manager will have real-time access to his or her current people KPIs on which to base decisions. All in all, it can thus be concluded that having high-quality and sufficient data, a high-quality IT infrastructure and a communication infrastructure that is both efficient and impactful is critical for an effective people analytics team. 
The KSAOs of people analytics staff: In the literature, the question of which KSAOs the experts of a people analytics team require is highly debated. Rasmussen and Ulrich (2015), for instance, argue that it is easier to teach HRM and related theories to people with strong statistical skills than the other way around, whereas Levenson (2005) cautions against hiring people with a lack of HRM knowledge because these people may draw the wrong conclusions about their findings, as not all HRM activities can be expressed in numbers. The current consensus on the topic is that for a people analytics team, strong HRM or psychological and statistical skills lead to the most effective teams. In the popular six-competency model of Andersen (2016), these two skills, in addition to storytelling, visualization skills, business acumen and strong data management skills, are seen as must-haves for people analytics teams. In 2017, Green added a seventh competency to this model, namely, change management, as he believes that teams should also ensure that their insights are successfully implemented in the organization. In addition to this, Davenport and Harris (2017) specifically mention stakeholder management capabilities in general as a core capability for a people analytics team. At least eight different KSAOs consequently appear to contribute to the effectiveness of a people analytics team.

\section{The types of products that a people analytics team should deliver to contribute to organizational performance}

Based on the literature, a people analytics team may offer three broad types of products to an organization in order to improve decision-making. These are (1) the development of employee monitoring tools, (2) organizational research, and through these, (3) establishing an evidencebased culture (e.g. Angrave et al., 2016; Marler and Boudreau, 2017).

Employee monitoring tools: Within the field of people analytics, one of the most common practices is to report basic information about personnel, such as the number of Full-Time Employee's (FTEs) and absenteeism ratios (Van den Heuvel and Bondarouk, 2017). The two most well-known employee monitoring tools are dashboards and scorecards (Angrave $e$ t al., 2016; Marler and Boudreau, 2017), which often contain historical data (Angrave et al., 2016), survey scores and benchmarking information (Davenport and Harris, 2017). According to Holsapple et al. (2014), these types of products can facilitate the identification of problems, share insights, facilitate decisions and spur stakeholders into action. A number of companies have reported that these descriptive statistics added the most value to their organizations (Van der Togt and Rasmussen, 2017). However, organizations have often not tested whether the concepts that they report truly have a relationship with important performance indicators (Levenson, 2005). Thus, it can be debated whether decision-makers are truly receiving the information they require to make the best decision possible. It therefore seems important to test the strategic relevance of the information contained in the reports to add the most value to an organization through this type of service (Bose, 2009).

\section{Organizational research}

Kaur and Fink (2017) and Levenson and Fink (2017) describe conducting organizational research as an important delivery for a people analytics team. Organizational research can be defined as the "studies or experiments conducted to address a specific, one-off organizational question" (Kaur and Fink, 2017, p. 15). By carrying out such research, organizations have, for instance, examined what the most important predictors are of team satisfaction, collaboration and performance for their specific organization (e.g. Google's project Aristotle). The benefit of this organization-specific research is that one can investigate the topics most relevant to an organization (Kaur and Fink, 2017), and the results can provide contextualized, specific insights. To conduct any organizational research, a people analytics team must first develop

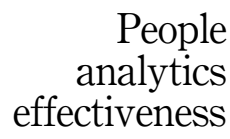


$\underset{7,2}{\text { JOEPP }}$

analytical models. Three types of analytical models can be distinguished in the literature, with three unique purposes. The first is the behavioral model, which uses existing data to establish causal relationships between predictors and the desired outcomes (Levenson, 2005). This type of model could, for instance, be used to determine the KSAOs that are related to high performance, which a recruiter may then use to make hiring decisions. The second model is the predictive model, which utilizes existing data to predict future outcomes (Levenson, 2005). In comparison to the previous model, this model may predict which of the applicants is most likely to become a high performer. The third model, a prescriptive model, also uses existing data but prescribes to decision-makers the action they should take (Davenport and Harris, 2017). In this case, the model would tell a recruiter which applicant he or she should hire. Specific to predictive and prescriptive models is that they can make use of machine learning and artificial intelligence (AI) (Guenole et al., 2017; Halo, no date). Machine learning and AI are both designed to autonomously identify patterns in large, complex bodies of data, such as text analysis (i.e. natural language processing). However, although AI is far more efficient than a human at analyzing text, it has also been reported to lack in accuracy (Kaur and Fink, 2017). In addition, Van den Heuvel and Bondarouk (2017) state that machine learning models do not necessarily consider causal relationships when making their predictions. Therefore, they indicate that this type of model building can best be done with many variables and when studying complex relationships. However, this does not come without risk, as the data analyst may no longer understand why a certain prediction or prescription is made by the model (e.g. Amazon's recruitment algorithm that discriminated against women (Dastin, 2018)). It can consequently be argued that the creation of the three analytical models and the exact methods used by these models should be considered based on their specific use-case. After all, each model is likely able to improve people's decisions in their own way and is thus capable of adding value to an organization. Therefore, an effective people analytics team is capable of not only building all three different models but also selecting the most appropriate model for the organizational question at hand.

\section{Establishing an evidence-based culture}

One of the most important goals of the analytical function is to establish a culture in which (personnel's) decisions are being made based on analytics and data (e.g. Davenport and Harris, 2017). As the core task of a people analytics team is to analyze and share data-driven insights about employees, that team is a vital element in the establishment of this culture. However, as Guenole et al. (2017) stress, an evidence-based culture also helps to ensure that stakeholders act on the insights provided by a people analytics team instead of ignoring them. This point is supported by Davenport and Harris (2017), who state that a culture in which stakeholders actively search for, understand, use and act on the insights provided by people analytics helps to make those teams prosper and grow. After all, once the use of data and analytics becomes more common practice, stakeholders will likely value their outputs more, which also further increases the power and reputation of the team. As such, the establishment of an evidence-based culture will likely directly influence the effectiveness and added value of people analytics teams.

Aside from delivering relevant, high-quality and data-driven products, Davenport and Harris (2017) state that support from senior management is crucial to establish an evidencebased culture. In particular, support from the CEO, senior management and senior leaders serving as role models are mentioned as critical success factors. The reason for this is that once senior managers push for the use of analytics, the attitude and mindset of other stakeholders, and particularly their subordinates, may change as well. It consequently appears that both the people analytics team and senior management are crucial factors in establishing an evidence-based culture. 


\section{The stakeholders of a people analytics team and the way in which they should be managed}

In the literature, stakeholder groups are classified in numerous ways. Davenport and Harris (2017) distinguish the various stakeholders of a team by their functional role, whereas Guenole et al. (2017) group stakeholders based on the relationship they have with their people analytics team (e.g. customers, gatekeepers and those impacted by the results). As membership in these groups is not mutually exclusive (Guenole et al., 2017), and since different stakeholders within the same group likely have varying interests and needs (e.g. line managers and employees are grouped in the same category), we have opted for classification in functional groups. We therefore discuss the following groups as the main stakeholders of a people analytics team: (1) HR professionals, (2) management (senior management and line management), (3) employees and their representatives and (4) other analytical teams (e.g. Guenole et al., 2017; Van den Heuvel; Bondarouk, 2017).

\section{HR professionals}

As people analytics teams generally focus on generating insights into the workforce, HR professionals are often seen as the most important stakeholders of a people analytics team. For HR professionals, people analytics allows them to demonstrate the impact of their initiatives on business outcomes (Mondore et al., 2011) and to combine their intuition with objective data and analytical insights to make better decisions (Rasmussen and Ulrich, 2015). In summary, people analytics can be seen as "HR (analytics) for HR" (Guenole et al., 2017), and HR professionals may therefore be the ones who could potentially benefit the most from having an effective people analytics team. However, as stated in the introduction, HR professionals are often not attracted to data and appear to be uncertain about how to utilize it (e.g. Angrave et al., 2016; Marler and Boudreau, 2017; Rasmussen and Ulrich, 2015), which hinders the adaptation of people analytics and limits the value a company can gain from analytical insights (Levenson, 2011; Marler and Boudreau, 2017). To solve these issues, an often-noted recommendation for effectively involving this stakeholder group is to educate its members on analytics and make it part of their DNA (Green, 2017; Minbaeva, 2018). Moreover, the following have been recommended: sharing success cases, focusing on topics that are truly relevant for HR professionals, analyzing HR practices and metrics associated with business issues and performance and ensuring that HR professionals become part of the analytical process from the beginning (Guenole et al., 2017). It can consequently be concluded that HR professionals are not only some of the most important stakeholders of a people analytics team but also a group that requires special attention from the team to become truly successful. As people analytics can only be effective when stakeholders act on the insights gleaned from it, successfully managing HR professionals seems crucial for a successful people analytics team.

\section{Senior management}

As mentioned previously, the support of senior management is seen as an enabler of an effective people analytics team. While senior management can help highlight the importance of people analytics to other stakeholders (Davenport and Harris, 2017; Smeyers 2016 as cited in Green, 2017; Guenole et al., 2017), it also comprises important customers for the team. By providing senior managers with relevant insights and recommendations, they can tackle pressing organizational issues and execute the business strategy (Van den Heuvel and Bondarouk, 2017; Van der Togt and Rasmussen, 2017). To do this successfully, having regular conversations with them is recommended to understand their needs and share the insights and recommendations in a way that can be easily understood, communicated and acted upon (Guenole et al., 2017; Van der Togt and Rasmussen, 2017). Moreover, by paying

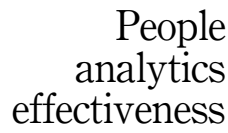


JOEPP
7,2 special attention to the "so what" question, Van der Togt and Rasmussen (2017) argue that stakeholders, such as senior and line management, can be spurred into action. This is crucial for effective people analytics teams, as they can add value to an organization only when their insights and recommendations are acted upon.

\section{Line management}

While HR professionals and senior management often create the overall personnel policies, line managers are generally tasked with their implementation and execution (e.g. Boxall et al., 2007; Wright and Nishii, 2012). Guenole et al. (2017) consequently argue that line managers are the ones affected by the findings of a people analytics team as they are likely to be the ones tasked to act on those findings (e.g. provide a salary increase to top performers to decrease voluntary attrition). In line with this, Levenson (2005) argues that line managers, similarly to HR professionals, should be the ones who own the change process - not a people analytics team - after people analytics provides them with the required insights. Van den Heuvel and Bondarouk (2017) argue, however, that many line managers have difficulty making decisions based on data. In particular, they report that line managers "find it hard to understand, accept and adopt the application of analytics in decision making" (Van den Heuvel and Bondarouk, 2017, p. 14). Guenole et al. (2017) argue that this resistance makes sense when line managers are asked to give up their decision-making responsibilities to a statistical model that tells them, for instance, who to hire, promote or fire. To get these stakeholders on board, Guenole et al. (2017) provide four useful tips. First, a people analytics team must understand what issues line managers are facing and how analytics may help them tackle these issues. Second, why, how and to what degree an analytical project may help line managers in their job must be explained well. Third, feedback should be gathered from line managers after the completion of an analytical project in order to learn how to improve. Fourth, past successful analytical projects should be presented to line managers. It consequently appears that this group too requires a combination of education and product relevance to effectively make use of people analytics products.

\section{Employees and employee representatives}

The fourth main stakeholders of a people analytics team, namely, employees, are also affected by the insights and recommendations provided by a people analytics team (Green, 2017; Guenole et al., 2017). Therefore, it is argued that organizations should be fully aware of the legal and moral obligations they have to their employees and conduct research that provides value to the organization as well as to the employees (Green, 2017; Mondore et al., 2011). Guenole et al. (2017) add to this that especially projects with potential positive or negative effects for a certain group of people should be carefully thought through to avoid a situation in which a group of employees may be damaged or alienated. For instance, while an analysis may suggest that employees with certain demographical characteristics react less to a salary increase than others, it would likely cause issues if this group was the only one to be excluded from a salary increase. After all, the employees who were excluded might feel unfairly treated, and the employees who were included may feel unfairly favored.

Employees may also be a potential risk for a people analytics team, because they own their data and privacy is becoming increasingly important (Green, 2017; Van den Heuvel and Bondarouk, 2017). As a result of the dependency on personnel data, Green (2017) warns that people analytics initiatives can be undermined if employees decide against sharing their data or provide irrelevant or untruthful data. To address this issue, Guenole et al. (2017) provide a number of recommendations. These are to be open with employees about how their data is being used, to ask for feedback about where additional analyses are needed and to demonstrate the benefits that people analytics projects have for them. The authors argue that 
the latter may also result in an increased willingness of employees to share their data with the people analytics team in the future (Guenole et al., 2017). Thus, for a people analytics team to be effective in the long term, it appears that the relationship between employees and their representatives needs to be carefully managed by the team.

\author{
People \\ analytics \\ effectiveness
}

\section{Other analytical teams}

Rasmussen and Ulrich (2015) argue that collaborating with other analytical teams can be beneficial for sharing knowledge, analytical models and techniques. They point out that this is especially beneficial to people analytics teams, considering that they are the newest type of analytical team and may thus lack expertise compared to the other analytical teams. Given that other analytics teams often also work with people data, there seems to be ample opportunity to benefit from their expertise indeed. This is also illustrated in one of the case studies described by Guenole et al. (2017) in which a people analytics lead believed that employees should be treated, and thus analyzed, in the same way as customers. He consequently applied customer segmentation to the workforce to determine what actions to take for different employee groups in order to improve the employee experience. Another benefit of teaming up with other analytical teams is that people analytics can benefit from their data definitions and data (Rasmussen and Ulrich, 2015), which can also increase the credibility of the findings of a people analytics team (Guenole et al., 2017). By teaming up with financial analytics, for instance, a people analytics team can link profitability to employee survey data, which in turn can potentially help HR to make a stronger case for investing in new HR initiatives, as illustrated by the case study of Van De Voorde et al. (2010).

\section{The elements of a people analytics team's proper governance structure to safeguard compliance and achieve legitimacy}

The main ingredient that a people analytics team requires for its analysis is data about its workforce. However, to protect people's privacy, a proper governance structure must be in place that is compliant with related legislation (such as the General Data Protection Regulation [GDPR] in Europe). Next, we focus on data governance (data management and ethics), governance of the people analytics function (organizational positioning, reporting structure, internal team structure and delivery channels) and finally building and maintaining social legitimacy.

\section{Data governance}

In recent years, data governance has become more important than it was in the past (Guenole et al., 2017) due to the increased need to comply with data privacy legislation, such as the GDPR in Europe, and employees' increased concerns about privacy. As data governance involves all activities related to the management of data and the ethical questions that surround it (Davenport and Harris, 2017; Guenole et al., 2017), we discuss each of these topics below.

\section{Data management}

Proper data management is seen as a must-have capability of any people analytics team to keep the trust of employees and to comply with the law (Van den Heuvel and Bondarouk, 2017; Van der Togt and Rasmussen, 2017). Therefore, procedures and rules should be in place with regard to how data should be managed, maintained and stored (Davenport and Harris, 2017). Aspects that should be considered by the team in this respect are anonymization, storage duration, storage location, data security, data access, data format and data 
$\mathrm{JOEPP}_{7,2}$

212 maintenance (Davenport and Harris, 2017; Guenole et al., 2017; Regulation (EU), 2016; Van der Laken, 2018). With regard to these topics, a people analytics team should be aware of the organization-specific agreements as well as the laws of the country in which it is operating (Guenole et al., 2017). In Europe for instance, many of these elements are regulated by law (e.g. GDPR). As a consequence, laws are in place that state, for example, that personal data should not be stored longer than necessary (Regulation(EU), 2016) and that data should be dealt with confidentially (i.e. accessed on a need-to-know basis) (Vegt, 2017). Due to the complexity of these topics, Guenole et al. (2017) recommend that a people analytics team should collaborate with specialized professionals, such as a data privacy officer. Professionals in this function have the task of ensuring that people's data is processed in compliance with the GDPR (European Data Protection Supervisor, no date), which is mandatory for any country operating in Europe. Therefore, to adhere to the law and be seen as legitimate, having the correct rules and procedures in place with regard to data management can be seen as another important pre-requisite for a team to work with people data and thus be effective.

\section{Ethics}

Guenole et al. (2017) define data ethics as the fundamental legal and moral principles about right and wrong related to the governance of data. Batistic and van der Laken (2019) argue that ethical considerations are even more important for an analytics team than adhering to legalization and privacy standards. They argue that this is especially the case when dealing with big data and predictive analytical models as they can, for instance, lead to self-fulfilling prophecies and bias (Batistič and van der Laken, 2019; Herschel and Miori, 2017), such as the aforementioned example in which gender discrimination was accidently included in the selection algorithm. Van der Laken (2018) explains that the ethical side to people analytics is also important because simply adhering to the law may not always be sufficient. As an example, he argues that although employees in Europe are required to give consent for their employer to analyze their data, employees may not feel as though they have the choice to refuse. Being mindful of data ethics is also in line with the recommendation of Mackaluso (as cited in Guenole et al., 2017), who argues that even if an analysis is possible, it does not make it automatically right to do so. For instance, although analyzing health data might be tempting, it should be considered carefully if an employer truly wants to start steering employees toward "good" (e.g. healthy) behavior. Moreover, as it is unclear what "good" behavior is, this would grant employers much influence over an employee's life (Van der Laken, 2018). Therefore, Van der Laken (2018) recommends careful consideration of the purpose of an analytical project beforehand, and Guenole et al. (2017) suggest partnering with HR to ensure correct usage of the data. In summary, for stakeholders to see a people analytics team and its project as legitimate, and for the team's effectiveness, it is important that the team is seen as mindful of ethical concerns in addition to adhering to the law.

\section{Governance of the people analytics function}

Following the HR government and risk management kaleidoscope (Farndale et al., 2010), we believe that people analytics as a function should consider its structure, delivery channels, the governance and control of its products and the monitoring of these products (Farndale et al., 2010) to safeguard compliance. In the next sections, we discuss the following internal governance ingredients: organizational positioning, the reporting structure, the internal team structure of a people analytics team and the delivery channels.

\section{Organizational positioning}

With regard to the organizational structure, two prevalent views are presented in the literature (Guenole et al., 2017). According to the first view, people analytics should be placed 
inside the HR function as a center of excellence $(\mathrm{CoE})$. This center refers to a team that provides "leadership, best practices, research, support and training" on a certain topic, such as people analytics (Guenole et al., 2017, p. 208). According to Van den Heuvel and Bondarouk (2017), a possible benefit of placing people analytics within the $\mathrm{CoE}$ is that it may lead to a close collaboration with HR. This can be advantageous considering the previously mentioned importance of this stakeholder. In addition, Levenson (2005) argues that a $\mathrm{CoE}$ within HR is required because HRM-specific theoretical and statistical knowledge is required to add value through people analytics. The second view argues that a people analytics team would benefit more from being placed outside of HR and together with other analytical teams (e.g. Rasmussen and Ulrich, 2015). As previously mentioned, collaborating with other analytical teams is considered to be beneficial due to the possibility of sharing expertise, analytical models and techniques and data. With regard to the latter, Rasmussen and Ulrich (2015) emphasize that only when personnel data is combined with data from other analytical fields, new insights will be born. As a result, the "so what" question, in which people analytics insights are translated to financial consequences (Levenson and Fink, 2017) and strategy (Minbaeva, 2018), may also become easier to answer. This is also what appears to be happening in practice as a number of people analytics practitioners have reported having a great impact on business issues while being part of the broader analytics department (Guenole et al., 2017).

\section{Reporting structure}

Although scholars do not agree on the best organizational positioning of a people analytics team, there is overall agreement about the importance of reporting to senior management. In particular, many scholars argue that a people analytics lead should directly report to the HR director (Smeyers 2016, as cited in Green, 2017; Guenole et al., 2017). According to Green (2017), this is crucial for three reasons. First, due to the HR director's unique position in an organization, he or she understands the organization's key people issues that the team can contribute to. Second, the HR director has the seniority to grant the team the required access to the right business leaders. Third, even in light of controversial analytical insights, the HR director has the influence to ensure that they are acted upon.

\section{Internal team structure}

As clarified in the section on the KSAO's of a people analytics team, an effective people analytics team has many different KSAOs. In practice, a people analytics lead's main responsibility is to effectively manage experts with different backgrounds and expertise, while also ensuring that the team and its projects are successfully navigated throughout the business (Guenole et al., 2017). To do so successfully, that lead might choose to split his or her team into specialized sub-teams. Kaur and Fink (2017), for instance, reported that a little more than half of the companies they interviewed split their team into reporting and analytics teams. The reason for this decision is to protect the resources of analytical experts from the ever increasing demands for HR-related reports (Kaur and Fink, 2017). Moreover, while reporting and analytics could technically be placed in different parts of an organization, Kaur and Fink (2017) also found benefits to centralizing reporting and analytics in one team. For instance, analytics needs to have access to the data of reporting and also needs to be able to control the data reporting it is generating (Kaur and Fink, 2017). As mentioned before, reporting can also benefit from analytics by, for instance, reporting on KPIs that have a known link with organizational performance. Therefore, it appears that although creating sub-teams within a people analytics team can increase its overall added value, keeping these different sub-teams relatively close together within an organization is even more important to achieve effective coordination and collaboration.

\section{People analytics effectiveness}

213 


\section{JOEPP 7,2}

\section{Delivery channels}

According to Paauwe (2004) and the HR government and risk management kaleidoscope (Farndale et al., 2010), a people analytics team should consider how and to whom the products of the team should be delivered. Apart from issues related to the management of stakeholders (see above), it should be noted that legal and ethical considerations should also be taken into account in terms of the delivery channel. The GDPR, for instance, states that organizations should process personal data with integrity and confidentiality, which generally means that people analytics teams are not allowed to deliver insights or data to stakeholders that will allow any individuals to be identified, unless the individuals consented to this beforehand (Regulation(EU), 2016). This means that, practically, the team should consider, for instance, which demographics to report, the granularity of its reports, the sensitivity of the insights and the purpose for which the data was collected. Moreover, this can also mean that after an engagement survey, some line managers in a team with high response should receive team insights, whereas a line manager with a team of only three respondents should not receive the same report due to the risk of personal identification. This will likely have consequences for the delivery structure and especially the capabilities of the IT and communication structure, which needs to be capable of reporting the insights on the correct level of granularity (i.e. team, department, business unit), while preserving the agreed upon anonymity.

\section{Governance of external social legitimacy}

The social legitimacy of an organization, and thus also a people analytics team, can be viewed through an internal lens (i.e. micro) as well as an external lens (i.e. macro). According to Paauwe and Farndale (2017, p. 101), legitimacy is based on "relational rationality," which ".....refers to establishing sustainable and trustworthy relationships with both internal and external stakeholders." As we have already mentioned how a people analytics team should approach internal stakeholders, in this section we focus on the external stakeholders the team requires to be perceived as legitimate. We specifically focus on (1) trade unions and employee representatives and (2) collaboration with external parties.

\section{Unions and employee representatives}

As unions and employee representatives aim to protect the rights and interests of employees, these stakeholders can be concerned about people analytics' usage of employee data. Especially in countries with a strong legislative basis for works councils, the agreement of a council may be required before a people analytics team is allowed to access and analyze certain data (Guenole et al., 2017). Van den Heuvel and Bondarouk (2017) mention that only within a few "progressive" organizations, the people analytics team collaborates with unions and workers councils, which will benefit the social legitimacy of the team both internally and externally.

\section{External parties}

Various scholars suggest that teaming up with external parties, such as consultancy firms and universities (e.g. Angrave et al., 2016; Cascio and Boudreau, 2011), can be beneficial to a people analytics team and will add to its prestige and trustworthiness. The rationale for this is that consultants and academics can bring deep (behavioral analyst) expertise that can help take a people analytics team to the next level (Angrave et al., 2016; Levenson, 2005). By partnering up with externals, companies may be able to showcase successful analytics projects to their stakeholders more quickly as a result and thereby accelerate the credibility of their analytics team (Guenole et al., 2017) and thus the social legitimacy of people analytics. 
The People Analytics Effectiveness Wheel@

Based on the previous overview, it is clear that there is not one ingredient that solely determines whether a people analytics team will be successful. Instead, multiple ingredients seem to be required to have the "enablers," "products," "stakeholders" or "governance structure" in place that will enhance decision-making and hence organizational performance. It also became clear that in addition to each ingredient contributing in its own unique way to the effectiveness of the team, a relationship also exists between the different ingredients and categories. For instance, while the internal governance structure of the team is important to achieve legitimacy, it will likely also affect where the team is based in the organization and hence the relationship with stakeholders. Based on the currently available literature, we can only state that the different ingredients are important for people analytics teams to be successful. However, we cannot yet determine what their relative importance is or what relationships among resources look like. As we believe that it is important to provide practitioners in particular with a comprehensive overview of how to equip their people analytics teams for success, we bundled the ingredients under their specific category in a framework: the People Analytics Effectiveness Wheel (Figure 1). This framework provides an easy-to-grasp overview of the categories and the ingredients critical to these categories, as identified in this article. The benefit of the current representation is that it does not touch on the cause or consequence of the ingredients or relative relevance, as this is still in need of further exploration by future research.

\section{Directions for future research}

By reviewing the existing literature on (people) analytics, we managed to identify which enablers, products, stakeholders and governance ingredients are critical for a people analytics team to be effective. Due to a lack of qualitative papers on the topic (Van der Laken, 2018), the most pressing question that remains is which processes should be in place to

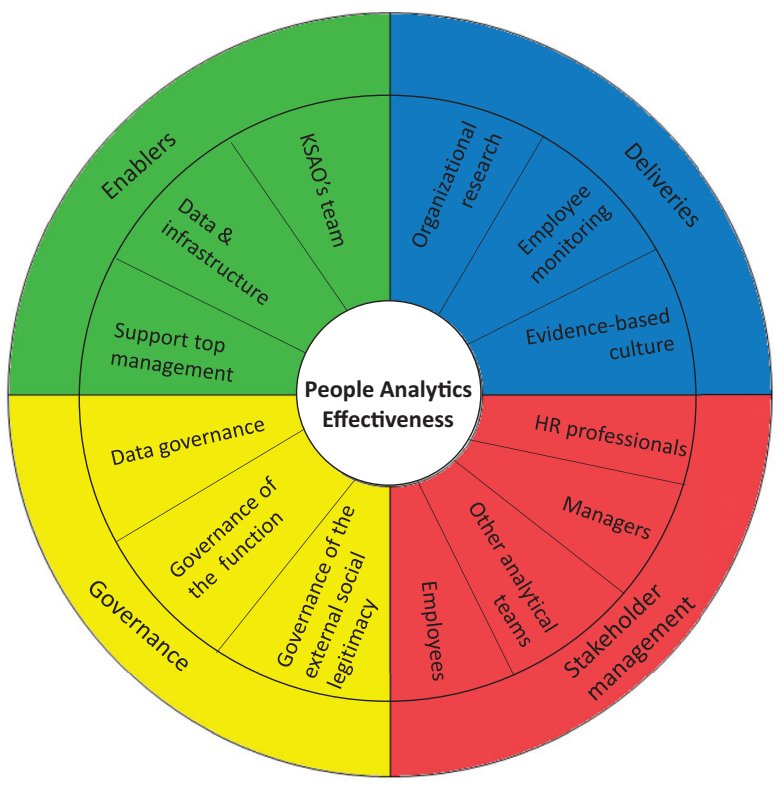

Figure 1. People Analytics Effectiveness Wheel C by the authors of this article 

$\underset{7,2}{\text { JOEPP }}$

\section{6}

successfully transform these ingredients into increased organizational performance. Now that the People Analytics Effectiveness Wheel can be used as a guideline, the next step is to uncover these processes in empirical research. Therefore, our primary recommendation is to carry out case studies and/or other forms of qualitative research with people analytics among a range of companies with varying degrees of success and experience in order to capture the underlying processes [1] between the different ingredients and categories of the framework. Furthermore, we recommend paying specific attention to the stakeholder and governance ingredients because we expect the most critical processes for a successful people analytics team to be present there. These ingredients will likely determine how internal and external stakeholders perceive a people analytics team, which will directly affect their willingness to take action on the analyses and insights the team delivers.

A second direction that future research could take is to study how the different ingredients may relate to and affect one another. For instance, while we believe that all ingredients are important for the success of a people analytics team, it may turn out that some ingredients are less critical than others. Moreover, we also believe that the ingredients may reinforce, substitute or undermine one another's value in certain situations. In particular, we expect that the four categories may "reinforce" one another when they are highly developed, boosting the effectiveness of a people analytics team. In the case of "substitution," we imagine that a team that lacks deep psychological skills may bring in this knowledge by collaborating well with its HR stakeholders. Finally, with respect to "undermining," it may be, for instance, that teams in the initial stages of developing a strong data governance structure lack the data to build (predictive) statistical models. This in turn also lowers the value of highly advanced statistical skills within the team, making these skills less valuable. We consequently recommend that researchers investigate the way in which ingredients affect one another's effectiveness and whether primary or secondary ingredients for success can be identified.

Third, although we believe that a people analytics team will be most effective when all of the different ingredients within the People Analytics Effectiveness Wheel are addressed accordingly, the inclusion of all the ingredients most likely requires a large number of (financial) resources and data, as well as a large analytics department. However, this is not to say that a small company will not benefit from conducting people analytics as well. As research has primarily focused on large companies that are generally at the forefront of people analytics (e.g. Kaur and Fink, 2017), little is known about the success factors for people analytics in small- and medium-sized firms thus far. We imagine they would use standardized analyses tools, join forces with similar companies or conduct research projects in collaboration with academic scholars to reap the benefits of people analytics. To ensure that they are not left behind, we recommend future research to explore the way in which small- and medium-sized organizations can execute people analytics effectively.

\section{Conclusion}

In this paper, we have identified and discussed the key ingredients that are required to establish an effective people analytics team based on the existing people analytics and business intelligence literature. This led to the development of our People Analytics Effectiveness Wheel, which can serve as an initial point of departure for enhancing decisionmaking and contributing with people analytics to organizational performance. From an academic point of view, our framework can be used as a heuristic device to explore the ingredients and processes that should be in place for a people analytics team to be successful. Exploratory follow-up research could also investigate how the different ingredients relate to one another and what their relative importance is. Furthermore, from a practitioner's point of view, our framework can act as a guideline for organizations that are considering how to set up their people analytics function. Finally, for organizations that already have a team, our 
framework can help them assess the different ingredients in terms of their quality, risk analysis, interrelationships and areas for improvement in order to increase their effectiveness.

\section{Notes}

1. We are currently working on such research in which multiple (advanced) people analytics teams and their stakeholders have been interviewed across the globe.

\section{References}

Andersen, M.K. (2016), "Six must-have competencies in a world-class analytics team", available at: https://mortenkamp.com/2016/06/06/six-must-have-competencies-in-a-world-class-analytics- team, (accessed 31 March 2020).

Andersen, M.K. (2017), "Human capital analytics: the winding road", Journal of Organizational Effectiveness: People and Performance, Vol. 4 No. 2, pp. 133-136.

Angrave, D., Charlwood, A., Kirkpatrick, I., Lawrence, M. and Stuart, M. (2016), "HR and analytics: why HR is set to fail the big data challenge", Human Resource Management Journal, Vol. 26 No. 1, pp. 1-11.

Barton, D. and Court, D. (2012), "Making advanced analytics work for you", Harvard Business Review, Vol. 90 No. 10, pp. 78-83.

Batistič, S. and van der Laken, P. (2019), "History, evolution and future of big data and analytics: a bibliometric analysis of its relationship to performance in organizations", British Journal of Management, Vol. 30 No. 2, pp. 229-251.

Bersin, J. (2012), "New research: BigData in HR as huge opportunity", available at: https://joshbersin. com/2012/04/new-research-bigdata-in-hr-as-huge-opportunity, (accessed 31 March 2020).

Bose, R. (2009), "Advanced analytics: opportunities and challenges", Industrial Management and Data Systems, Vol. 109 No. 2, pp. 78-83.

Boudreau, J. and Cascio, W. (2017), "Human capital analytics: why are we not there?", Journal of Organizational Effectiveness: People and Performance, Vol. 4 No. 2, pp. 119-226.

Boxall, P.F., Purcell, J. and Wright, P.M. (2007), The Oxford Handbook of Human Resource Management, Oxford University Press on Demand, Oxford, Melbourne.

Cascio, W. and Boudreau, J. (2011), Investing in People: Financial Impact of Human Resource Initiatives, 2nd ed., Pearson Education, Inc., Upper Saddle River, NJ.

Dastin, J. (2018), Amazon Scraps Secret AI Recruiting Tool that Showed Bias against Women, Reuters, available at: https://www.reuters.com/article/us-amazon-com-jobs-automation-insight/amazonscraps-secret-ai-recruiting-tool-that-showed-bias-against-women-idUSKCN1MK08G, (accessed 31 March 2020).

Davenport, T. and Harris, J. (2017), Competing on Analytics: Updated, with a New Introduction: The New Science of Winning, Harvard Business Review Press, Boston, MA.

Edwards, M.R. and Edwards, K. (2019), Predictive HR Analytics: Mastering the HR Metric, 2nd ed., Kogan Page Ltd, London.

Eurostat (2013), "Glossary: full-time equivalent (FTE)", available at: https://ec.europa.eu/eurostat/ statistics- explained/index.php?title=Glossary:FTE, (accessed 31 March 2020).

Farndale, E., Paauwe, J. and Boselie, P. (2010), "An exploratory study of governance in the intra-firm human resources supply chain", Human Resource Management, Vol. 49 No. 5, pp. 849-868.

Green, D. (2017), "The best practices to excel at people analytics", Journal of Organizational Effectiveness: People and Performance, Vol. 4 No. 2, pp. 137-144.

Guenole, N., Ferrar, J. and Feinzig, S. (2017), The Power of People: Learn How Successful Organizations Use Workforce Analytics to Improve Business Performance, Cisco Press, Indianapolis, IN. 
$\mathrm{JOEPP}_{7,2}$

Herschel, R. and Miori, V.M. (2017), "Ethics and big data", Technology in Society, Vol. 49, pp. 31-36.

Holsapple, C., Lee-Post, A. and Pakath, R. (2014), “A unified foundation for business analytics”, Decision Support Systems, Vol. 64, pp. 130-141.

Hota, J. and Ghosh, D. (2013), "Workforce analytics approach: an emerging trend of workforce management", AIMS International Journal, Vol. 7 No. 3, pp. 167-179.

Kaur, J. and Fink, A.A. (2017), Trends and Practices in Talent Analytics, Society for Human Resource Management (SHRM)-Society for Industrial-Organizational Psychology (SIOP) Science of HR White Paper Series, available at: https://www.shrm.org/hr-today/trends-and-forecasting/specialreports-and-expert-views/Documents/2017\%2010_SHRM-SIOP\%20Talent\%20Analytics.pdf (accessed 31 March 2020).

Levenson, A. (2005), "Harnessing the power of HR analytics", Strategic HR Review, Vol. 4 No. 3, pp. 28-31.

Levenson, A. (2011), "Using targeted analytics to improve talent decisions", People and Strategy, Vol. 34 No. 2, pp. 1-26.

Levenson, A. and Fink, A. (2017), "Human capital analytics: too much data and analysis, not enough models and business insights", Journal of Organizational Effectiveness: People and Performance, Vol. 4 No. 2, pp. 145-156.

Marler, J.H. and Boudreau, J.W. (2017), "An evidence-based review of HR Analytics”, International Journal of Human Resource Management, Vol. 28 No. 1, pp. 3-26.

Minbaeva, M. (Ed.) (2017), "Human capital analytics: why are not we there?", Journal of Organizational Effectiveness: People and Performance, Vol. 4 No. 2, pp. 110-118.

Minbaeva, D.B. (2018), "Building credible human capital analytics for organizational competitive advantage", Human Resource Management, Vol. 57 No. 3, pp. 701-713.

Mondore, S., Douthitt, S. and Carson, M. (2011), "Maximizing the impact and effectiveness of HR analytics to drive business outcomes", People and Strategy, Vol. 34 No. 2, pp. 20-27.

Paauwe, J. (2004), HRM and Performance: Achieving Long-Term Viability, Oxford University Press, Melbourne.

Paauwe, J. and Farndale, E. (2017), Strategy, HRM, and Performance: A Contextual Approach, 2nd ed., Oxford University Press, Melbourne.

Rasmussen, T. and Ulrich, D. (2015), "Learning from practice: how HR analytics avoids being a management fad", Organizational Dynamics, Vol. 44 No. 3, pp. 236-242.

Regulation(EU) (2016), "Regulation (EU) 2016/679 of the European parliament and of the council", available at: https:/eur-lex.europa.eu/legal-content/EN/TXT/?uri=uriserv:OJ.L_.2016.119.01. 0001.01.ENG\&toc=OJ:L:2016:119:FULL, (accessed 31 March 2020).

Sierra-Cedar, I. (2018), Sierra-Cedar 2018-2019 HR Systems Survey White Paper, 21st Annual Edition, available at: https://www.sierra-cedar.com/wp-content/uploads/Sierra-Cedar_2018-2019_HR SystemsSurvey _WhitePaper.pdf, (accessed 31 March 2020).

Trkman, P., McCormack, K., De Oliveira, M.P.V. and Ladeira, M.B. (2010), "The impact of business analytics on supply chain performance”, Decision Support Systems, Vol. 49 No. 3, pp. 318-327.

Van De Voorde, K., Paauwe, J. and Van Veldhoven, M. (2010), "Predicting business unit performance using employee surveys: monitoring HRM-related changes", Human Resource Management Journal, Vol. 20 No. 1, pp. 44-63.

Van den Heuvel, S. and Bondarouk, T. (2017), "The rise (and fall?) of HR analytics", Journal of Organizational Effectiveness: People and Performance, Vol. 4 No. 2, pp. 157-178.

Van der Laken, A.P. (2018), Data-driven Human Resource Management: The Rise of People Analytics and its Application to Expatriate Management, Dissertation.

Van der Togt, J. and Rasmussen, T.H. (2017), “Toward evidence-based HR”, Journal of Organizational Effectiveness: People and Performance, Vol. 4 No. 2, pp. 127-132. 
Vegt, L. (2017), "How will the GDPR change the approach to security?", available at: https:// eugdprcompliant.com/will-gdpr-change-approach-security, (accessed 31 March 2020).

Wright, P. and Nishii, L.H. (2012), "Strategic human resource management and organizational behaviour: exploring variance as an integrating framework", in Paauwe, J., Guest, D.E. and Wright, P. (Eds.), HRM and Performance: Achievements and Challenges, John Wiley \& Sons Ltd, Chicester, Sussex, pp. 97-110.
People analytics effectiveness

\section{Corresponding author}

Tina Peeters can be contacted at: t.peeters@tilburguniversity.edu 